\title{
Blind Spot: A Braille Patterned Novel Multiplex Lateral Flow Immunoassay Sensor Array for the Detection of Biothreat Agents
}

E. Randal Hofmann' Charles Davidson, Hsiu Chen, Melody Zacharko, Jay E. Dorton Gary K. Kilper, Carcie Graves, Aleksandr E. Miklos, Katherine Rhea, Joe Ma, Bruce G. Goodwin, Shanmuga Sozhamannan* 


\section{Supplementary Figures}

\section{Figure S1.}

Blind Spot concept. Alphabets and corresponding modified Braille spotting patterns. The alphabets and the corresponding spots in modified Braille (normal Braille font contains three rows of 2 spots each and in the modified Braille all six positions are in a row) is shown below on the left. The different pathogens/agents or analytes are given a letter code according to the preference of the assay developer and the corresponding detector antibodies are spotted in those specific patterns on the LFI strip. There are $2^{\wedge} 6$ possibilities for creating patterns using the 6 spots scheme. PC- positive control.

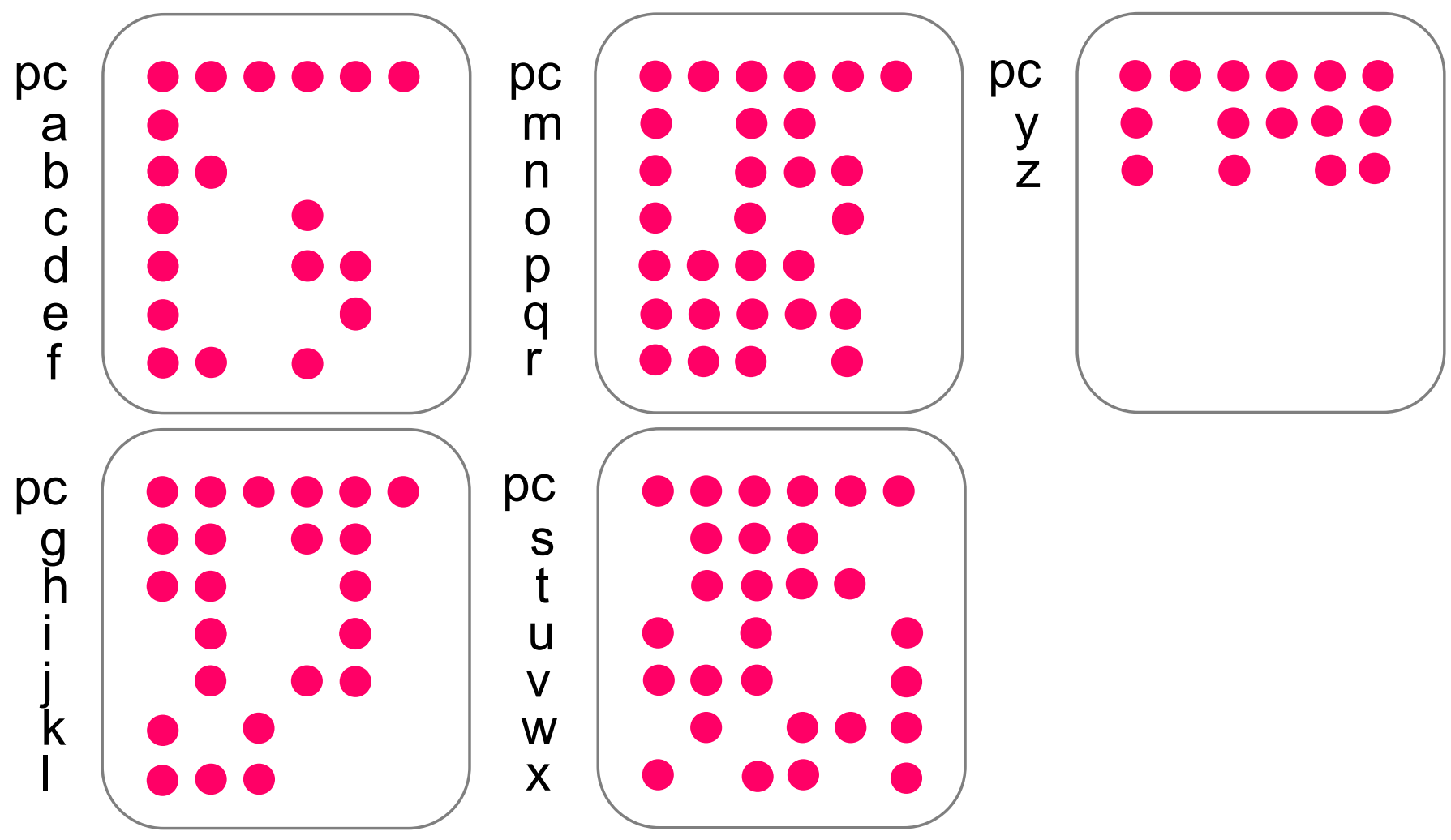




\section{Figure S2.}

Individual toxoid visual results. Toxoids were tested individually at indicated concentrations. The diluent used is included a blank control for comparison.
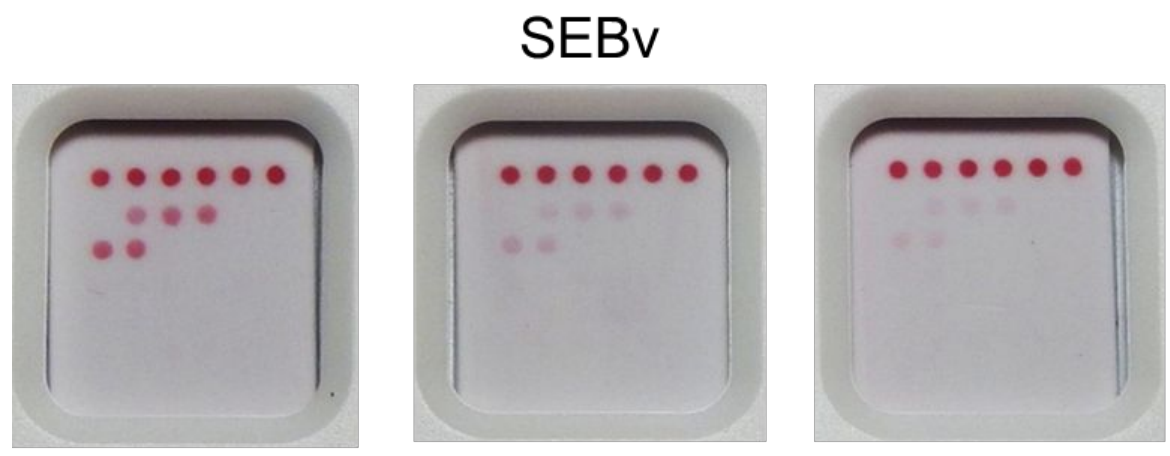
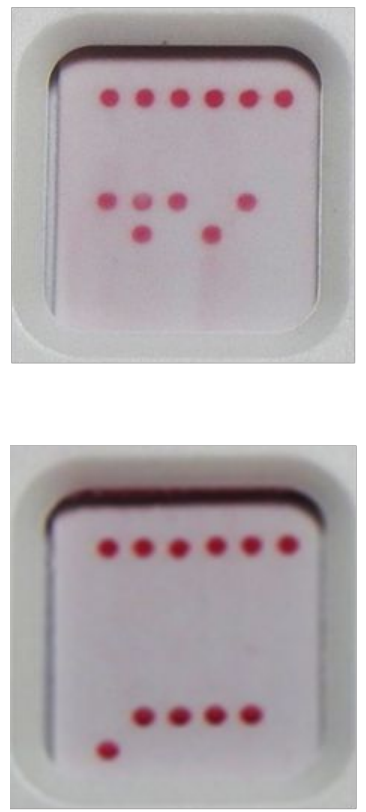

100

\section{RicA}

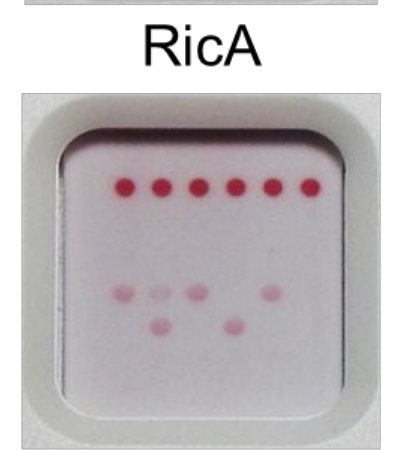

\section{BotA}

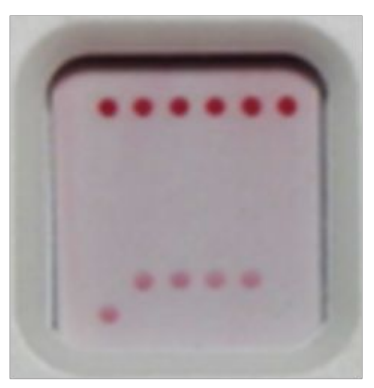

10

$\mathrm{ng} / \mathrm{mL}$

\section{Diluent}
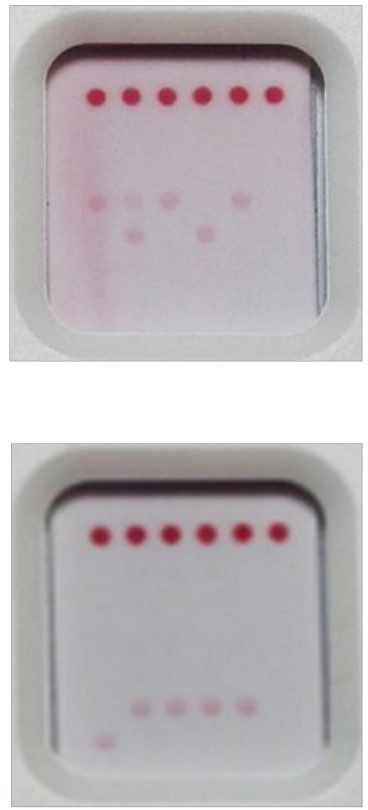

5

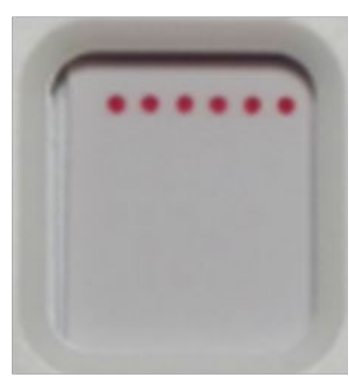




\section{Supplementary Methods}

\section{Description of MACAW}

MACAW stands for Modular Automated Colorimetric Analysis Widget and consists of a portable reader based on a Raspberry Pi Zero W equipped with the Camera Module V2. A custom $3 \mathrm{D}$ printed case houses the Raspberry $\mathrm{Pi}$, camera, LED light source, and OLED screen. A $3 \mathrm{D}$ printed removable sample stage was designed to align the LFI assay cassettes and block out ambient room lighting. The device was used with custom Python code to collect images in triplicate of the LFI assays under test.

Images of the LFI assays were post-processed on a laptop using MACAW image processing tools. The image processing tools perform three steps: (1) recognize, align, and crop the Blind Spot ticket image, (2) detect the spots on the ticket, and (3) extract RGB averages of the spots and background ticket areas. The alignment step identifies the LFI window and then looks for the control line and other high-contrast spots. These spots are used to infer the orientation angle and crop boundaries needed to align the Blind Spot ticket. In the detection step, statistical anomaly detection (thresholding the Mahalanobis distance of each pixel against the white ticket background) identifies possible spots. The ticket is broken up into grid tiles and within each tile the most likely area(s) are identified as the spot. Morphological processing and area constraints are applied; in the case that no spot is identified a circle in the center of the tile is used instead. The extraction step computes statistics such as mean and covariance of each spot as well as the surrounding background (empty) ticket areas. Figure $S_{3}$ shows the 
results of each of these image-processing steps. The RGB averages for the spots and the empty tile regions are then subject to a detection algorithm for identifying the presence of the Braille letter patterns. Also utilized is a confidence metric designed to combat occasional streaks (relatively large colored areas spanning multiple grid tiles) caused by unbound gold nanoparticles. The confidence metric is $1-f$, where $f$ is the area fraction computed by ratioing the area within the tile that is part of an object that straddles grid boundaries to the total spot area in the tile. For a typical spot that is located at the center of the grid tile, $f$ will equal zero and the confidence will equal one. For a "spot" that is solely part of a streak that crosses grid boundaries, the fraction $f$ will equal one and the confidence will be zero.
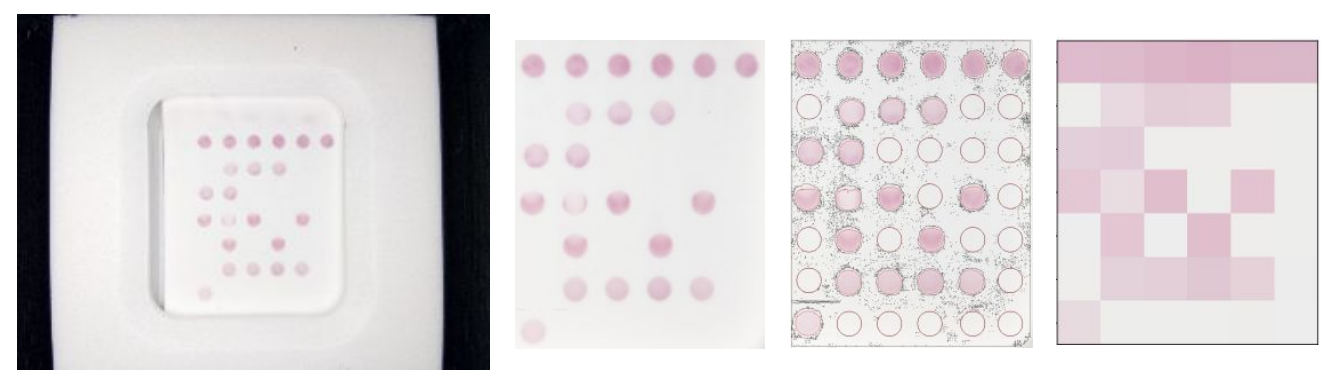

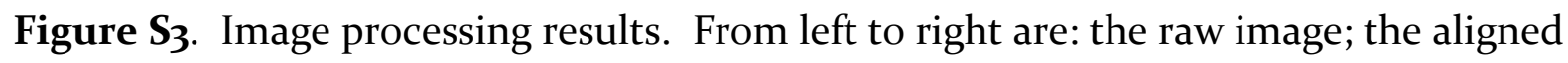
and cropped ticket image; the ticket image with spot detection contours overlaid in red and empty (background) region contours overlaid in black; and the extracted RGB averages for the spot in each tile. The spot averages are analyzed further for identification of Braille letter patterns on the Blind Spot ticket.

The algorithm for identification of Blind Spot Braille patterns is briefly summarized here. The algorithm analyzes the RGB data for expected response patterns 
estimating three quantities that contribute to the detection decision: signal intensity, pattern distance or shape, and probability. The idea is that if the response is not intense enough, nor sufficiently close to the expected Braille letter patterns, nor statistically confident enough, then no positive identification should be made. Each metric is subject to its own decision threshold, and all thresholds must be met to issue a positive identification.

The first step is to preprocess the spot averages converting them to relative reflectance to the empty (white) ticket background. Let $\boldsymbol{\mu}_{1}$ and $\boldsymbol{\mu}_{\mathrm{o}}$ denote RGB averages for the spots and empty tile regions, respectively. Each is a matrix of size 3-by-42 holding RGB values for the 42 grid tiles, where we unwrap the grid in a row-major order. The matrix of relative reflectance values for the spots is given by $\mathbf{R}=\boldsymbol{\mu}_{1} / \boldsymbol{\mu}_{0}$, where the division is performed element-by-element. The empty tile areas are also converted to reflectance units using the global average of the empty ticket areas; these values are used to compute a noise covariance, $\mathbf{C}_{\mathrm{o}}$. A signal covariance, $\mathbf{C}_{1}$, is found from the control line tiles of $\mathbf{R}$. Each covariance is computing in a weighted fashion using the confidence metric from the image-processing step. These covariance matrices serve as an estimate of the variability of the ticket background and of the control line response, respectively.

The second step estimates the intensity of each individual spot relative to the control line. The reflectance average for the control line, weighted by confidence is computed: $\mathbf{t}=\left(\sum \mathrm{c}_{\mathrm{i}} \mathbf{R}_{i}\right) /\left(\sum \mathrm{c}_{\mathrm{i}}\right)$ for $i=1,2, \ldots, 6$, where $c_{i}=1-f_{i}$. This is an estimate of the "target" reflectance, i.e, it is the expected reflectance (or color) of a spot, and is used convert the observed spot reluctances to a relative intensity. This is done through linear 
unmixing treating the vectors $\mathbf{t}$ and $\mathbf{b}=\left[\begin{array}{ll}1 & 1\end{array}\right]^{T}$ as endmembers. The unconstrained least squares solution for the $i^{\prime}$ th tile is given by $\mathbf{W}_{i}=\mathbf{A}^{\#} \mathbf{R}_{i}=\left(\mathbf{A}^{T} \mathbf{A}\right)^{-1} \mathbf{A}^{T} \mathbf{R}_{i}$ where $\mathbf{A}$ is the 3-by-2 endmember matrix $[\mathbf{t} \mathbf{b}]$ and the solution vector $\mathbf{W}=\left[\mathrm{w}_{t} \mathbf{w}_{b}\right]$ givens the abundance fractions for the target and background, respectively. The $w_{t}$ value is the relative signal strength of the spot in the tile that is used going forward. Generally, it is a value between o and 1 but since we chose not to enforce non-negativity or sum-to-one constraints in the linear mixture model values may slightly exceed this nominal range. A plot of the wild type values for each tile for the ticket considered in Figure $S_{3}$ is shown in Figure $S_{4}$.

The next steps in the algorithm compares the 42-element relative signal strength vector, $\mathbf{w}_{t}$, against the expected signal patterns implied by the Braille letters. Three decision metrics are derived: signal magnitude, signal shape, and a probability or likelihood score. In this paragraph, we discuss the first metric, signal magnitude, in which the goal is to estimate the strength or intensity of each group of spots that comprise the response for each toxin. A pattern indicator matrix $\mathbf{P}$ encodes the expected signals, where $\mathbf{P}_{i}$ is a length 42 vector giving 1 where a response is expected for the ith pattern and o otherwise. For the Blind Spot ticket analyzed here the patterns are the control line $\left(i=1, \mathbf{P}_{1}=1\right.$ for the first 6 tiles $) \operatorname{SEB}\left(i=2, \mathbf{P}_{2}=1\right.$ on tiles 8-10, 13, 14) ricin $(i=3$, $\mathbf{P}_{3}=1$ on tiles 19-21, 23, 26, 28), and botulinum toxin ( $\mathrm{i}=4, \mathbf{P}_{4}=1$ on tiles $\left.32-35,37\right)$. A complementary pattern indicator matrix, $\mathbf{K}$, encodes with a $\mathbf{1}$ the empty tiles on the set of rows that contribute to the i'th pattern. For instance, for the SEB pattern $(i=2)$ that occurs across rows 2 and 3 on the Blind Spot ticket, $\mathbf{K}_{2}=1$ on tiles $7,11,12,15-18$ and is zero elsewhere. It is also convenient to define the union of the two pattern vectors, $\mathbf{G}=\mathbf{K} \| \mathbf{P}$, 
where the || symbol represents the logical OR operator. Thus, $\mathbf{G}_{i}$ identifies all grid tiles (positive or negative) that contribute to the toxin (i.e., rows 2-3 in the case of SEB, rows 45 in the case of ricin, and rows 5-6 in the case of BOT), $\mathbf{P}_{i}$ identifies those tiles that should have a response if the toxin is there, and $\mathbf{K}_{i}$ identifies those tiles that should not have a response. To account for confidence, each pattern vector in $\mathbf{P}$ and $\mathbf{K}$ are multiplied by the confidence vector, so that non-zero values in the pattern matrices take the confidence value for the corresponding tiles. The average signal strengths (projection magnitudes) for the ith pattern is given by $S_{i}=\left(\mathbf{w}_{t} \cdot \mathbf{P}_{i}\right) /\left\|\mathbf{P}_{i}\right\|^{2}$. By construction, $S_{1}=1.0$, whereas the strengths of the other patterns are a fraction of the control line strength. Projection magnitudes in the null space of each pattern toxin pattern $(\mathrm{i}=2,3,4)$, are computed similarly, $N_{i}=\left(\mathbf{w}_{t} \cdot \mathbf{K}_{i}\right) /\left.|| \mathbf{K}_{i}\right|^{2}$. For the control line, $N_{1}$ is set to o by assumption. An ideal toxin response would result in $S_{i}=1$ (average signal strength as intense as the control line) and $N_{i}=\mathrm{o}$ (no response on tiles that should not have responded). Sufficient signal strength is determined by thresholding the value of $\mathrm{Si}$. The $S_{i}$ values are shown in Figure S4 as colored horizontal lines.

The distance decision metric is how similar the observed signal pattern is to the expected pattern for the corresponding set of Braille letters. Digitization thresholds $\gamma_{i}$ are applied to the $\mathbf{w}_{t} \in \mathbf{G}_{i}$ to create estimated signal pattern matrices $\mathbf{P}^{\prime}$. A weighted Hamming distance (computed as a fraction) between $\mathbf{P}_{i}{ }_{i}$ and $\mathbf{P}_{i}$ serves as the second decision metric. The distance is zero if the digitized signal pattern matches the expected pattern exactly, and is equal to 1 if it matches the complement. Sufficient signal shape is achieved if the distance is less than a tolerance. 
The probability decision metric is related to how likely it would be to achieve a perfectly digitized signal (one matching the expected pattern) based on chance. Confidence-weighted standard deviations of the $\mathbf{w}_{t}$ values on each pattern $\mathbf{P}_{i}$ and each complementary pattern $\mathbf{K}_{i}$, denoted $\sigma_{S i}$ and $\sigma_{N i}$, respectively. We assume a Gaussian model such that $\mathbf{w}_{t} \in \mathbf{P}_{i} \sim \mathrm{N}\left(S_{i}, \sigma_{S i}{ }^{2}\right)$ and $\mathbf{w}_{t} \in \mathbf{K}_{i} \sim \mathrm{N}\left(N_{i}, \sigma_{N i}{ }^{2}\right)$. Given the digitization threshold $\gamma \mathbf{i}$, we ask how likely it would be for elements of $\mathbf{w}_{t} \in \mathbf{P}_{i}$ to exceed $\gamma$ i while simultaneously all elements of $\mathbf{w}_{t} \in \mathbf{K}_{i}$ failed to exceed the threshold. The probability is given by $\left(1-F_{S}\right)^{p}\left(F_{N}\right)^{\mathrm{n}}$ where $F_{S}$ denotes the cumulative distribution function (CDF) corresponding to the distribution $\mathrm{N}\left(S_{i}, \sigma_{S i}{ }^{2}\right)$ and $F_{N}$ denotes the CDF for $\mathrm{N}\left(N_{i}, \sigma_{N_{i}}{ }^{2}\right)$. The exponents $p$ and $n$ are the effective number of nonzero elements in the $\mathbf{P}_{i}$ and $\mathbf{K}_{i}$, respectively, and strictly speaking should be indexed with a subscript letter $i$, that is, $p_{i}=$ $\sum \mathbf{P}_{i}$ and $n_{i}=\sum \mathbf{K}_{i}$. As this probability exceeds 0.5 and approaches 1 it is less and less likely that we could have achieved the expected digitization of the signal based on chance.

Default thresholds for the three decision metrics were chosen as follows. The threshold for sufficient signal strength of each pattern was set to 1.5 standard deviations above the background (complementary pattern) mean. The default distance threshold was set to be $1 /\left(n_{i}+p_{i}+1\right)$, which means that there must be at least as many agreements in the two vectors as confidently measured tiles. In the case were the confidence values are all equal to 1 , this is a stringent threshold that requires the distance to be zero, but this becomes less stringent as the number of confidently measured tiles in a pattern is reduced. Finally, the default threshold for the the probability metric is to exceed o.5. In 
the results section we discuss modifications tot he default thresholds to improve performance based on observed behaviors of the Blind Spot tickets responses.

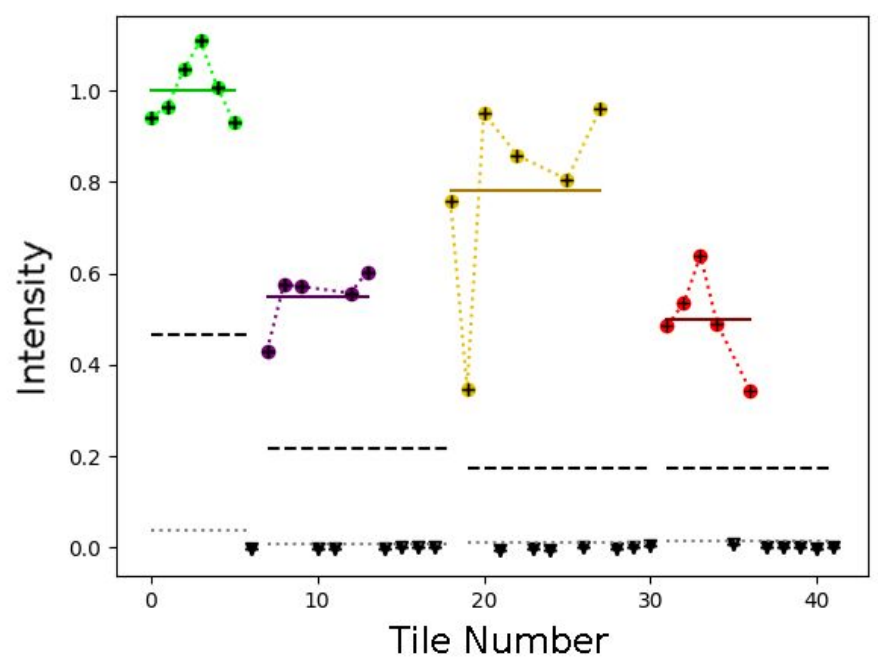

Figure $\mathbf{S}_{\mathbf{4}}$. Spot intensity values $\left(\mathbf{w}_{t}\right)$ for the 42 grid tiles corresponding to the LFI image

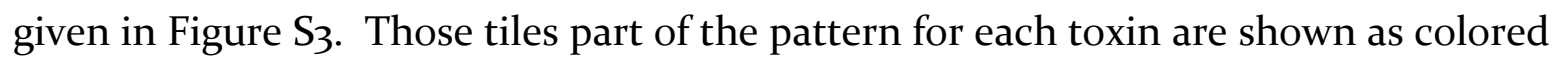
circles: control line (green), SEB (purple), ricin (yellow), and BOT (red). The horizontal line of the same color gives the average projection magnitude, $S_{i}$, for each pattern. The tiles that should not have a response are black. Dashed black horizontal lines show the digitization thresholds applied. For this case, all signal magnitudes are sufficiently strong, the shape of the response patterns matches the expected, and the result is statistically significant. This ticket would be correctly identified as a mixture containing all three toxins. 